\title{
Dyspnoea, lung function and CT findings 3 months after hospital admission for COVID-19
}

\author{
Tøri Vigeland Lerum ${ }^{1}$, Trond Mogens Aaløkken ${ }^{2,3}$, Eivind Brønstad ${ }^{4,5}$, \\ Bernt Aarli, ${ }^{6,}$, Eirik Ikdahl ${ }^{8}$, Kristine Marie Aarberg Lund ${ }^{9}$, \\ Michael T. Durheim ${ }^{3,10}$, Jezabel Rivero Rodriguez ${ }^{2}$, Carin Meltzer ${ }^{2}$, \\ Kristian Tonby 3,11 , Knut Stavem (1) $^{3,8,12}$, Ole Henning Skjønsberg ${ }^{1,3}$, \\ Haseem Ashraf ${ }^{3,13,14}$ and Gunnar Einvik $3,8,14$
}

@ERSpublications

3 months after discharge, a quarter of COVID-19 survivors have reduced gas diffusion capacity and persistent parenchymal opacities. ICU treatment is associated with persistent parenchymal opacities, but not with dyspnoea or reduced diffusing capacity. https://bit.ly/2J3xH4x

Cite this article as: Lerum TV, Aaløkken TM, Brønstad E, et al. Dyspnoea, lung function and CT findings 3 months after hospital admission for COVID-19. Eur Respir J 2021; 57: 2003448 [https://doi.org/10.1183/ 13993003.03448-2020].

ABSTRACT The long-term pulmonary outcomes of coronavirus disease 2019 (COVID-19) are unknown. We aimed to describe self-reported dyspnoea, quality of life, pulmonary function and chest computed tomography (CT) findings 3 months following hospital admission for COVID-19. We hypothesised outcomes to be inferior for patients admitted to intensive care units (ICUs), compared with non-ICU patients.

Discharged COVID-19 patients from six Norwegian hospitals were enrolled consecutively in a prospective cohort study. The current report describes the first 103 participants, including 15 ICU patients. The modified Medical Research Council (mMRC) dyspnoea scale, the EuroQol Group's questionnaire, spirometry, diffusing capacity of the lung for carbon monoxide $\left(D_{\mathrm{LCO}}\right), 6$-min walk test, pulse oximetry and low-dose CT scan were performed 3 months after discharge.

mMRC score was $>0$ in $54 \%$ and $>1$ in $19 \%$ of the participants. The median (25th-75th percentile) forced vital capacity and forced expiratory volume in $1 \mathrm{~s}$ were $94 \%(76-121 \%)$ and $92 \%(84-106 \%)$ of predicted, respectively. $D_{\text {LCO }}$ was below the lower limit of normal in $24 \%$ of participants. Ground-glass opacities (GGO) with $>10 \%$ distribution in at least one of four pulmonary zones were present in $25 \%$ of participants, while $19 \%$ had parenchymal bands on chest CT. ICU survivors had similar dyspnoea scores and pulmonary function as non-ICU patients, but higher prevalence of GGO (adjusted OR 4.2, 95\% CI 1.1-15.6) and lower performance in usual activities.

3 months after admission for COVID-19, one-fourth of the participants had chest CT opacities and reduced diffusing capacity. Admission to ICU was associated with pathological CT findings. This was not reflected in increased dyspnoea or impaired lung function.

This article has an editorial commentary: https://doi.org/10.1183/13993003.04423-2020

This article has supplementary material available from erj.ersjournals.com

The full protocol has been published on www.clinicaltrials.gov (NCT04535154). An anonymised data file will be shared through the website of the journal upon publication.

Received: 9 Sept 2020 | Accepted: 18 Nov 2020

Copyright OERS 2021. This version is distributed under the terms of the Creative Commons Attribution NonCommercial Licence 4.0. 


\section{Introduction}

The lower airways and lungs are the primary targets for the severe acute respiratory syndrome coronavirus 2 (SARS-CoV-2). The majority of patients requiring hospital admission for coronavirus disease 2019 (COVID-19) have respiratory symptoms such as cough and dyspnoea, in addition to signs of impaired lung function with varying degrees of hypoxaemia [1]. These symptoms are associated with widespread ground-glass opacities (GGO) on chest computed tomography (CT) scans and chest radiography [2, 3]. $\sim 15-30 \%$ of hospital-admitted COVID-19 patients develop severe respiratory failure and acute respiratory distress syndrome (ARDS), which necessitate admission to intensive care units (ICUs) and possibly mechanical ventilation $[1,4,5]$. According to the World Health Organization (WHO), the fatality rate from COVID-19 is $1-10 \%$, depending on age and underlying comorbidities [6].

As the COVID-19 pandemic represents a new disease, the long-term pulmonary outcomes in survivors of COVID-19 are unknown. Evidence from other coronavirus pneumonias, such as SARS and Middle East Respiratory Syndrome (MERS), suggests that impaired lung function and parenchymal opacities persist only in a minority of patients not having required mechanical ventilation [7]. However, in patients developing ARDS, as many as $11-45 \%$ have impaired lung function and persistent infiltrates on radiography after 10-12 months [8]. In order to identify and manage potential long-term sequelae of COVID-19, more research on the natural course of the disease is warranted [9]. Early reports of survivors following hospital admission for COVID-19 show reduced diffusing capacity, total lung capacity, exercise capacity or abnormal chest CT scan in almost 50\% after 1 month [10].

In the current study, we assessed patient-reported dyspnoea, lung function, quality of life (QoL) and parenchymal opacities in chest CT scans 3 months after hospital admission for COVID-19 in a prospective, consecutive Norwegian cohort of patients with or without ICU treatment.

\section{Materials and methods Design and participants}

Patient-Reported Outcomes and Lung Function After Hospital Admission for COVID-19 (PROLUN) is a multicentre prospective cohort study performed in six major hospitals in Norway. The study was approved by the regional ethics committee for South-Eastern Norway (no. 125384), by data protection officers at each participating centre, and registered to ClinicalTrials.gov (NCT04535154).

Patients aged $>18$ years who had been admitted for $>8 \mathrm{~h}$ with a discharge diagnosis (International Statistical Classification of Diseases and Related Health Problems 10) of U07.1 (COVID-19, virus identified), U07.2 (COVID-19, virus unidentified) or J12.x (viral pneumonia, in combination with positive SARS-CoV-2 identification in nasopharyngeal swab) were considered for eligibility. Exclusion criteria included living outside the hospitals' catchment areas, inability to provide informed consent or participation in the WHO Solidarity Trial.

Eligible patients were invited by mail $\sim 6$ weeks after hospital discharge. Informed consent was obtained by return of a written signed consent form or through a secure digital consent form (Services for Sensitive Data (TSD), University of Oslo, Oslo, Norway). One telephone reminder was performed for nonrespondents.

In accordance with the study protocol, an interim report of the first 100 participants was planned. This number was achieved on June 24, 2020. The current study thus comprises all participants who had attended the 3-month follow-up visit by June 24, 2020 ( $\mathrm{n}=103)$.

\section{Data collection}

Participants returned to the respective hospitals' outpatient clinics for a 3-month follow-up visit. The median (25th-75th percentile) time between the hospital admission and the 3-month visit was 83 (73-90) days;

Affiliations: ${ }^{1}$ Dept of Pulmonary Medicine, Oslo University Hospital Ullevål, Oslo, Norway. ${ }^{2}$ Dept of Radiology and Nuclear Medicine, Oslo University Hospital Ullevål, Oslo, Norway. ${ }^{3}$ Institute for Clinical Medicine University of Oslo, Oslo, Norway. ${ }^{4}$ Thoracic Dept, St Olavs Hospital, Trondheim, Norway. ${ }^{5}$ Dept of Circulation and Medical Imaging. Faculty of Medicine and Health Sciences NTNU, Trondheim, Norway. ${ }^{6}$ Dept of Thoracic Medicine, Haukeland University Hospital, Haukeland, Norway. ${ }^{7}$ Dept of Clinical Science, University of Bergen, Bergen, Norway. ${ }^{8}$ Pulmonary Dept, Akershus University Hospital, Lørenskog. Norway. ${ }^{9}$ Dept of Infectious Diseases, Østfold Hospital Trust Kalnes, Grålum, Norway. ${ }^{10}$ Dept of Respiratory Medicine, Oslo University Hospital Rikshospitalet, Oslo, Norway. ${ }^{11}$ Dept of Infectious Diseases, Oslo University Hospital Ullevål, Oslo, Norway. ${ }^{12}$ Health Services Research Unit, Akershus University Hospital, Lørenskog, Norway. ${ }^{13}$ Dept of Diagnostic Imaging, Akershus University Hospital, Lørenskog, Norway. ${ }^{14}$ Shared last authors.

Correspondence: Gunnar Einvik, Pulmonary Dept, Akershus University Hospital, 1470 Lørenskog, Norway. E-mail gunnar.einvikamedisin.uio.no 
82 (73-90) days in the non-ICU group and 85 (81-90) days in the ICU group ( $p=0.090)$. The criteria for admission to ICU were similar across centres: inability to maintain a satisfactory pulse oximetric saturation $\left(S_{\mathrm{pO}_{2}}\right)$ through oxygen supplementation by nasal cannula or non-rebreather mask. In addition, the participants were assessed by an anaesthesiologist before transfer to the ICU.

\section{Self-reported dyspnoea}

The modified Medical Research Council (mMRC) dyspnoea scale, range 0-4, was used [11, 12]. This measure was not administered for the first 18 participants, or at St Olavs Hospital (Trondheim, Norway). However, for these participants, we performed additional analyses with the last value carried forward from self-reported mMRC 4-6 weeks prior to the visit.

QoL

The EuroQol Group's EQ-5D-5L questionnaire [13] was used to measure health-related QoL. It contains five items scored on an ordinal scale from 1 (no problems) to 5 (unable/extreme problems). This questionnaire was administered by mail or weblink 4-6 weeks prior to the visit. Scores were available for $88(89 \%)$ of the participants. EQ-5D index values were prepared using the crosswalk method with UK weights [14].

\section{Pulmonary function tests}

Spirometry was conducted to measure the forced vital capacity (FVC) and the forced expiratory volume in $1 \mathrm{~s}\left(\mathrm{FEV}_{1}\right.$ ) ( Jaeger MS-PFT Analyzer Unit, Höechberg, Germany and CareFusion type MasterScreen PFT, Yorba Linda, CA, USA; software SentrySuite V03.0.5; Vyaire Medical, Höechberg). The ratio of $\mathrm{FEV}_{1} / \mathrm{FVC}$ was calculated. Diffusing capacity of the lungs for carbon monoxide $\left(D_{\mathrm{LCO}}\right)$ and alveolar ventilation $\left(V_{\mathrm{A}}\right)$ were measured, and $D_{\mathrm{LCO}} / V_{\mathrm{A}}$ (transfer coefficient of the lung for carbon monoxide) was calculated. All procedures were executed according to the American Thoracic Society (ATS) and European Respiratory Society (ERS) guidelines $[15,16]$. The Global Lung Function Initiative (GLI) network reference values were used to calculate the percentage of predicted values, the lower limit of normal (LLN) and z-scores $[17,18]$. A 6-min walk test (6MWT) was performed according to ATS/ERS guidelines, with baseline $S_{\mathrm{pO}_{2}}$ measured by pulse oximetry on index fingers [19].

\section{Chest CT}

Low-dose, thin-section CT images were obtained in supine and prone positions, during breath-holding and deep inspiration. The same CT protocol, adjusted for the different CT scanners employed, was used for all examinations. The tube current settings were adjusted to each patient's weight, with low-dose references at $120 \mathrm{kVp}$, high pitch and shortest possible rotation time. For evaluation of lung parenchyma, we applied thin reconstructed slice thickness $(0.9-1.25 \mathrm{~mm})$, with a high spatial frequency kernel, and a softer kernel with thicker $(2-3 \mathrm{~mm})$ slices for mediastinal evaluation. Two experienced thoracic radiologists independently reviewed all images, blinded to the participants' clinical history. The degree of consensus was high. The presence, extent and distribution of interstitial findings were registered using nomenclature recommended by the Fleischner Society [20]. For the purpose of the current analysis, GGO and parenchymal bands were assessed. Findings were registered in four separate apicobasal zones of the lungs using anatomical landmarks in the mediastinum [21].

\section{Other clinical variables}

Baseline demographic characteristics (sex, age, height, weight, history of smoking), body mass index (BMI), comorbidities (diabetes or hypertension) and data from the COVID-19 hospital admissions were obtained from the electronic patient records. Clinical variables indicating the severity of COVID-19 were use of oxygen, admission to ICU, use of mechanical ventilation, the maximal levels of C-reactive protein (CRP) and D-dimer.

All collected data was stored in TSD, designed for storing and post-processing sensitive data in compliance with the Norwegian Personal Data Act and Health Research Act.

\section{Statistical analyses}

For continuous data, median and 25th-75th percentiles were reported in descriptive statistics. Group comparison was performed with Mann-Whitney U-tests or Chi-squared tests, as appropriate. For lung function variables the predicted value was calculated, reporting LLN and z-score.

Descriptive analyses of the cohort were considered important for this interim report. We also tested the hypothesis that participants admitted to the ICU would have more dyspnoea, lower lung function, lower QoL and more pathological CT findings than participants not admitted to the ICU. 
The main outcome measures were 1) $\mathrm{mMRC} \geqslant 1$; 2) $D_{\text {LCO }}$ below LLN; 3) >10\% GGO in at least one lung zone; and 4) the presence of parenchymal bands. Secondary outcomes were 6MWT distance, $S_{\mathrm{pO}_{2}}$, EQ-5D-5L scores and EQ-5D index. The association between COVID-19 severity indices and main outcomes were assessed by univariate logistic regression analyses. The association between ICU admission, pre-defined as the major indicator of COVID-19 severity, and the main outcomes were adjusted by multivariable analysis. Due to a limited number of participants, only a few independent variables were allowed: age and sex, except for $D_{\text {LCO }}$ below LLN, in which current/previous smoking versus never smoking was adjusted for. All statistical analyses were performed using Stata (version 16.1; StataCorp, College Station, TX, USA). A p-level $<0.05$ was considered statistically significant.

\section{Results}

Demographic and clinical characteristics from the hospital admission are shown in table 1. Several indices of COVID-19 severity were higher in participants admitted to ICU than in the non-ICU participants: length of stay, oxygen therapy, the maximum levels of CRP and D-dimer and the prevalence of bilateral lung densities. However, the demographic variables (sex, age, BMI and smoking status) were comparable between the groups. Hypertension was less commonly observed in those admitted to ICU than in non-ICU participants. The median (25th-75th percentile) time from symptom onset to admission were 7 (5-9) days and 9 (6-11) days in ICU and non-ICU participants, respectively ( $\mathrm{p}=0.125)$.

Table 2 presents self-reported dyspnoea, pulmonary function tests and results of chest CT scans in the whole group, as well as according to ICU admission subgroup during the hospital stay. Dyspnoea (mMRC score $>0)$ was reported by $37(54 \%)$ of the respondents, while $13(19 \%)$ reported mMRC score $>1 \quad(n=69)$. The prevalence of dyspnoea 3 months after discharge was similar between ICU and non-ICU participants (table 2). In sensitivity analyses including additional 23 participants with mMRC scores reported a few weeks earlier, the prevalence of mMRC score $>0$ and mMRC score $>1$ were $52(56 \%)$ and $22(24 \%)$, respectively $(n=93)$. For all pulmonary function tests, the majority of participants had values within the limits of normal. $\sim 10 \%$ had FVC or $\mathrm{FEV}_{1}$ below LLN, while $24 \%$ had significantly reduced $D_{\text {LCO }}$. Furthermore, patients admitted to ICU during hospital admission did not have reduced lung function, 6-min walk distance or oxygen saturation compared to those not admitted to ICU (table 2).

GGO were common; $24 \%$ of the participants had one or more lung zones with $\geqslant 10 \%$ presence of GGO. The presence of GGO was associated with age and CRP in univariable logistic regression analysis ( $p=0.009$ and $\mathrm{p}=0.001$, respectively) (supplementary table $\mathrm{S} 1$ ). The crude prevalence of GGO was not significantly different between ICU and non-ICU participants; however, after adjustment for age and sex, the odds of

TABLE 1 Clinical characteristics during admission for coronavirus disease 2019 (COVID-19)

\begin{tabular}{|c|c|c|c|c|}
\hline & Total & ICU admission & No ICU admission & p-value \\
\hline Subjects & 103 & 15 & 88 & \\
\hline \multicolumn{5}{|l|}{ Demographic data } \\
\hline Age years & $59(49-72)$ & $52(50-59)$ & $61(49-74)$ & 0.116 \\
\hline Body mass index $\mathrm{kg} \cdot \mathrm{m}^{-2}$ & $25.8(23.8-29.6)$ & $24.9(23.7-29.3)$ & $25.9(23.8-29.1)$ & 0.885 \\
\hline Current smoker & $3(3.4)$ & $0(0)$ & $3(4)$ & 0.216 \\
\hline History of hypertension & 35 (35) & 1 (7) & $34(40)$ & 0.017 \\
\hline History of diabetes & $8(8)$ & $0(0)$ & $8(9)$ & 0.599 \\
\hline \multicolumn{5}{|l|}{ COVID-19 hospital admission } \\
\hline Length of stay days & $6(3-11)$ & $17(12-25)$ & $5(3-9)$ & $<0.001$ \\
\hline Oxygen treatment & $67(66)$ & $15(100)$ & $52(59)$ & 0.003 \\
\hline ICU admission & $15(15)$ & $\mathrm{N} / \mathrm{A}$ & N/A & N/A \\
\hline Lowest level of lymphocytes $\times 10^{9}$ cells $\cdot L^{-1}$ & $0.9(0.6-1.4)$ & $0.8(0.6-1.0)$ & $1.0(0.6-1.5)$ & 0.129 \\
\hline
\end{tabular}

Data are presented as $\mathrm{n}, \mathrm{n}(\%)$ or median (25th-75th percentile) unless otherwise stated. ICU: intensive care unit; CRP: C-reactive protein; N/A: not applicable. 
TABLE 2 Pulmonary outcomes 3 months after hospitalisation

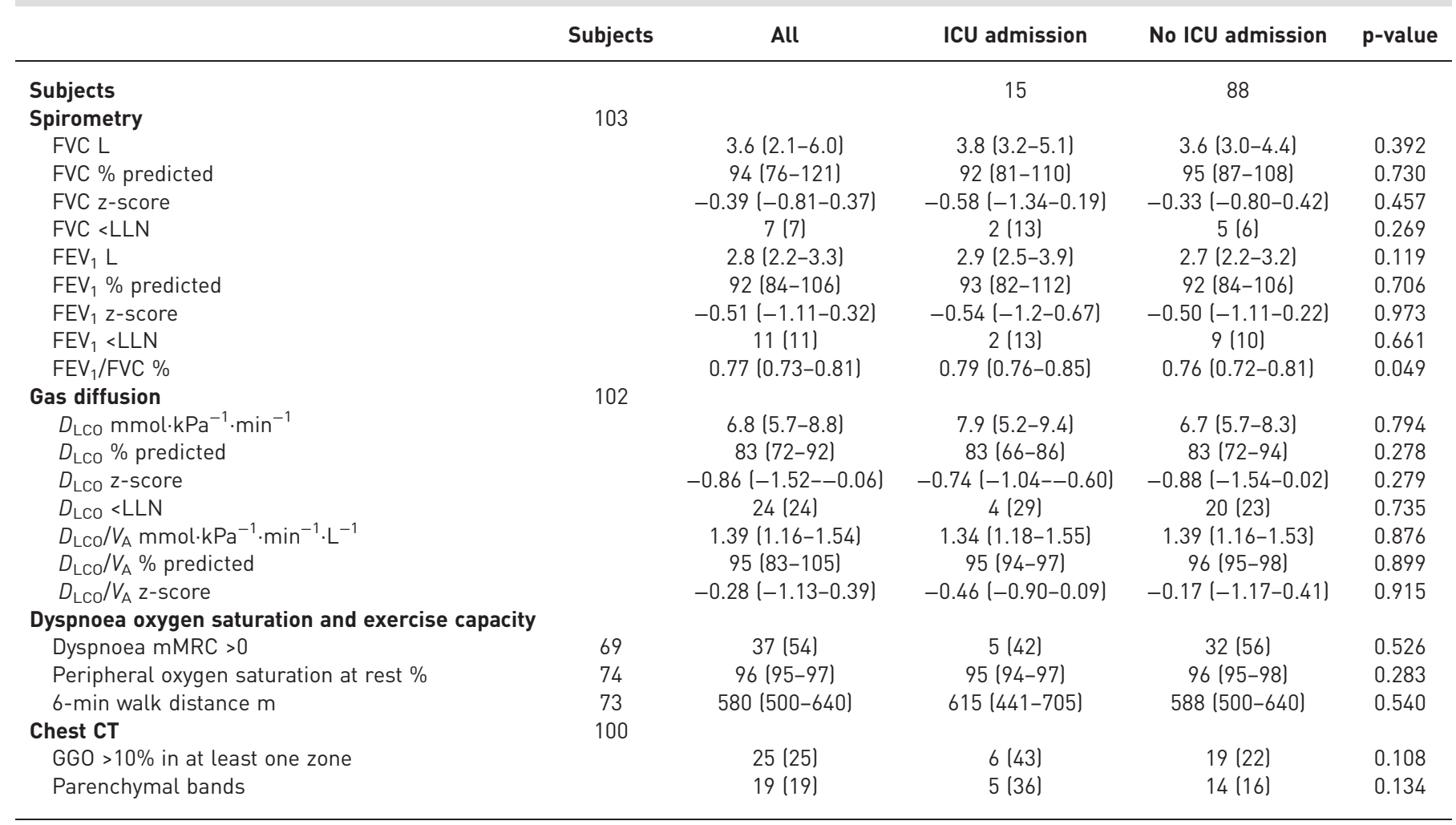

Data are presented as $\mathrm{n}$, median (25th-75th percentile) or $\mathrm{n}(\%)$, unless otherwise stated. ICU: intensive care unit; FVC: forced vital capacity; LLN: lower limit of normal; $\mathrm{FEV}_{1}$ : forced expiratory volume in $1 \mathrm{~s} ; D_{\mathrm{Lco}}$ : diffusing capacity of the lung for carbon monoxide; $V_{\mathrm{A}}$ : alveolar volume; mMRC: modified Medical Research Council dyspnoea scale; CT: computed tomography; GGO: ground-glass opacities.

having these pathological CT findings were significantly higher in participants admitted to ICU, compared to those not admitted to ICU (table 3). Parenchymal bands, indicating early progression to fibrosis, were found in one out of five participants. With regard to parenchymal bands, an association with CRP and

TABLE 3 Association of dyspnoea, gas diffusing capacity, ground-glass opacities (GGO) or parenchymal bands with admission to the intensive care unit (ICU)

\begin{tabular}{|c|c|c|c|}
\hline & Subjects $n$ & OR $(95 \% \mathrm{CI})$ & p-value \\
\hline Dyspnoea $^{\#}(\mathrm{mMRC}>0$ versus $\mathrm{mMRC}=0$ ) & 69 & & \\
\hline Male sex & & $0.39(0.14-1.08)$ & 0.069 \\
\hline Age per 10 years & & $0.81(0.57-1.14)$ & 0.231 \\
\hline ICU admission & & $0.67(0.18-2.50)$ & 0.553 \\
\hline Gas diffusing capacity ${ }^{\#}\left(D_{\mathrm{Lco}}<\mathrm{LLN}\right.$ versus $\left.D_{\mathrm{Lco}} \geqslant \mathrm{LLN}\right)$ & 102 & & \\
\hline History of smoking & & $1.56(0.61-4.03)$ & 0.356 \\
\hline ICU admission & & $1.54(0.42-5.61)$ & 0.517 \\
\hline GGO in chest $\mathrm{CT}^{\#}$ (>10\% GGO in $\geqslant 1$ zone versus none) & 101 & & \\
\hline Male sex & & $1.25(0.45-3.46)$ & 0.662 \\
\hline Age per 10 years & & $1.81(1.21-2.72)$ & 0.004 \\
\hline ICU admission & & $4.22(1.14-15.6)$ & 0.031 \\
\hline Parenchymal bands in chest $\mathrm{CT}^{\#}$ (yes versus no) & 101 & & \\
\hline Male sex & & $1.35(0.47-3.89)$ & 0.584 \\
\hline Age per year & & $1.19(0.81-1.74)$ & 0.376 \\
\hline ICU admission & & $2.99(0.83-10.8)$ & 0.093 \\
\hline \multicolumn{4}{|c|}{ 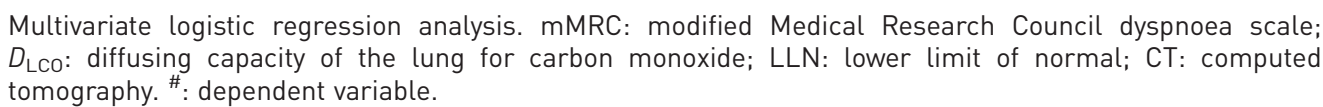 } \\
\hline
\end{tabular}



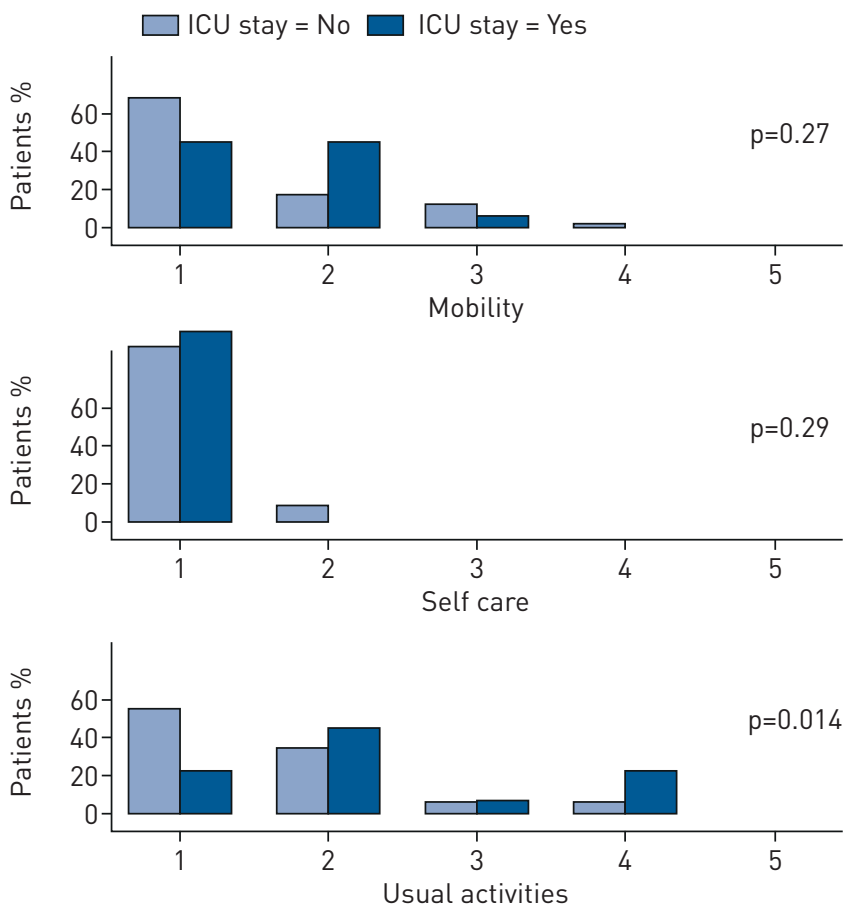

FIGURE 1 Distribution of EQ-5D-5L dimension scores for intensive care unit (ICU) (n=13) and non-ICU patients $(\mathrm{n}=75)$. 1: no problems, 2: slight problems, 3: moderate problems, 4: severe problems, 5: unable/extreme problems.
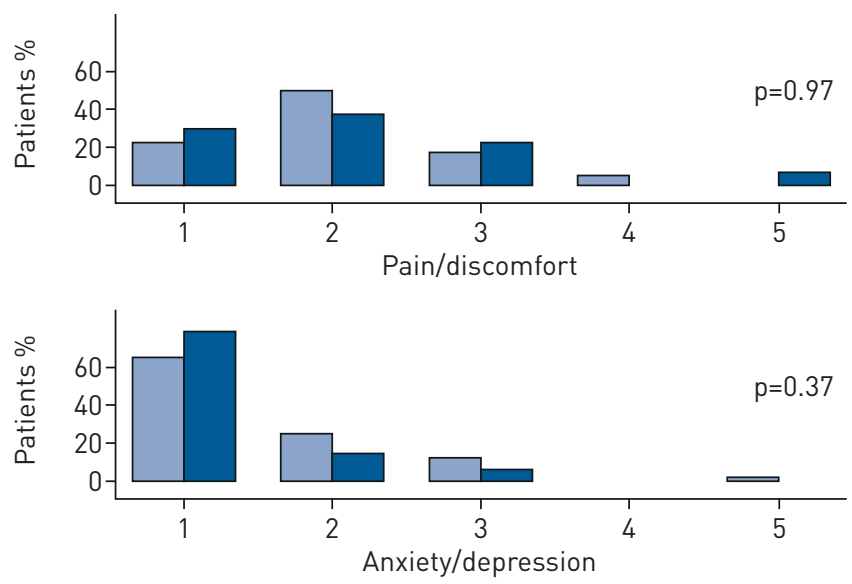

ventilator treatment was observed ( $\mathrm{p}=0.018$ and $\mathrm{p}=0.038$, respectively) (supplementary table S1). There was no difference between participants admitted to ICU and participants not admitted to ICU (table 3).

The scores on the five EQ-5D-5L items are shown in figure 1. Participants admitted to ICU had a higher median score on usual activities than participants admitted to regular wards only: 4 (25th-75th percentile $2-4)$ versus $2(1-2)$, respectively $(\mathrm{p}=0.014)$. The median \pm SD EQ-5D index scores were $0.61 \pm 0.23$ and $0.72 \pm 0.19$ for ICU and non-ICU patients, respectively $(\mathrm{p}=0.087)$.

\section{Discussion}

In this 3-month follow-up of a prospective cohort study of patients surviving hospital admission for COVID-19, approximately half of all participants had persistent dyspnoea on exertion, and one in four had reduced $D_{\mathrm{LCO}}$. Persistent GGO on CT scans were present in one-fourth of the participants, while one in five had parenchymal bands. Participants admitted to ICU during hospital admission had higher prevalence of persistent CT abnormalities and reported more problems in usual activities, but similar lung function and self-reported dyspnoea to those not admitted to ICU.

The favourable spirometry outcomes observed in this cohort were accompanied by a low prevalence of reduced peripheral oxygen levels and reduced exercise capacity, as indicated by the 6MWT. In total, our results indicate that development of chronic respiratory failure after 3 months is not common in survivors of COVID-19 hospital admission, including survivors of ICU admission. The degree of self-reported dyspnoea 3 months after hospital admission was not associated with prior ICU stay, which we used as a 
marker of COVID-19 severity. Dyspnoea is subjective and may be influenced by a number of other variables. The proportion of participants in our study with reduced diffusing capacity after 3 months was comparable to studies of SARS and MERS, and to other early reports in COVID-19 survivors [7, 10, 22, 23].

Persistent opacities in $\geqslant 10 \%$ of one or more lung parenchyma zones, as assessed by CT, were present in approximately one in four participants after 3 months. Compared with reports after 4 weeks of follow-up in another cohort [10], this finding suggests that COVID-19 related GGO may resolve without development of persistent fibrosis. However, some of these CT findings may persist and gradually develop into fibrotic changes, as reflected by the finding of parenchymal bands in one-fifth of our study population [24]. In a retrospective study of COVID-19 patients 8 weeks after hospital discharge, 25\% showed signs of early fibrosis [22]. This is consistent with findings in our cohort. We found that age was associated with persistent GGO in our material, which is consistent with findings regarding community-acquired pneumonia [25]. In addition, explorative analyses find the inflammatory marker CRP to be associated with both persistent GGO and parenchymal bands. Interestingly, parenchymal bands were also associated with ventilator treatment. However, it is still unknown if the persistent pathological CT findings will progress to symptomatic pulmonary fibrosis over time or if various treatments for COVID-19 may influence the long-term outcome of parenchymal opacities.

We found CT abnormalities after 3 months to be more common in participants admitted to ICU. Yet, there were no differences in lung function or diffusing capacity, $S_{\mathrm{pO}_{2}}$ or $6 \mathrm{MWT}$ distance between these participants and participants not admitted to ICU, despite ICU patients having more bilateral lung opacities on chest radiography and higher levels of markers of systemic inflammation during hospital admission. In addition, all patient-reported outcomes were similar between groups, except that more participants admitted to ICU reported an impaired ability to perform usual activities. If replicated in additional studies, the association between ICU admission, persistent pathological CT opacities and lower performance of usual activities may indicate that patients admitted to ICU warrant closer clinical follow-up than other patients admitted to hospital for COVID-19.

A strength of the study is the multicentre prospective design, where all survivors from hospitals covering a catchment area of 1.8 million residents were assessed for eligibility. The age and prevalence of ICU admission were similar between our sample and metadata from the Norwegian COVID-19 statistics (https://www.fhi.no/sv/smittsomme-sykdommer/corona). Yet, we cannot exclude participation bias. Participation in the WHO Solidarity trial was an exclusion criterion, but the hospitals in the current study started inclusion in Solidarity only towards the end of the inclusion period of the current study. Overall, we believe the study cohort to be representative for survivors of COVID-19 hospital admission in Norway. Finally, due to the limited sample size, the current study was not powered to explore associations between possible pathophysiological mechanisms and persistent dyspnoea, reduced diffusing capacity or parenchymal opacities.

\section{Conclusion}

In our Norwegian cohort, approximately half of all participants reported dyspnoea on exertion 3 months after hospital admission for COVID-19. The majority of participants had lung volumes within the reference limits, while one-fourth had reduced diffusing capacity. CT scans showed that one in four had persistent GGO, and one in five had parenchymal bands. ICU admission was associated with persistent CT abnormalities and reduced ability to perform usual activities, but not with dyspnoea, impaired lung function or reduced functional capacity, 3 months after discharge from the hospital.

Author contributions: G. Einvik conceived the study. The protocol was designed by T.M. Aaløkken, E. Brønstad, E. Ikdahl, M.T. Durheim, K. Stavem, O.H. Skjønsberg, H. Ashraf and G. Einvik in collaboration. Patient inclusion and data collection were performed by T.V. Lerum, E. Brønstad, B. Aarli, K.M.A. Lund, K. Tonby, K. Stavem, O.H. Skjønsberg and G. Einvik. T.M. Aaløkken, J.R. Rodriguez, C. Meltzer and H. Ashraf interpreted and analysed the CT findings. Statistical analyses were performed and analysed by T.V. Lerum, K. Stavem and G. Einvik. The first draft of the manuscript was written by T.V. Lerum, O.H. Skjønsberg, K. Stavem and G. Einvik. All authors contributed with considerable critical review of the manuscript and approval of the final version.

Conflict of interest: T.V. Lerum has nothing to disclose. T.M. Aaløkken has nothing to disclose. E. Brønstad has nothing to disclose. B. Aarli reports personal fees for lectures and advisory board work from AstraZeneca, personal fees for lectures from GlaxoSmithKline, Novartis, Boehringer Ingelheim and Chiesi Pharma, outside the submitted work. E. Ikdahl has nothing to disclose. K.M.A. Lund has nothing to disclose. M.T. Durheim reports grants and personal fees from Boehringer Ingelheim, personal fees from Roche and AstraZeneca, outside the submitted work. J.R. Rodriguez has nothing to disclose. C. Meltzer has nothing to disclose. K. Tonby has nothing to disclose. K. Stavem has nothing to disclose. O.H. Skjønsberg has nothing to disclose. H. Ashraf reports grants from Boehringer Ingelheim, during the conduct of the study. G. Einvik reports grants from Boehringer Ingelheim, during the conduct of the study; personal fees for consultancy from AstraZeneca AB, outside the submitted work. 
Support statement: The pulmonary and radiological departments at Akershus University Hospital have received unrestricted grants from Boehringer Ingelheim for funding of CT scans and study coordination in the current study. Funding information for this article has been deposited with the Crossref Funder Registry.

\section{References}

1 Zhou F, Yu T, Du R, et al. Clinical course and risk factors for mortality of adult inpatients with COVID-19 in Wuhan, China: a retrospective cohort study. Lancet 2020; 395: 1054-1062.

2 Kang Z, Li X, Zhou S. Recommendation of low-dose CT in the detection and management of COVID-2019. Eur Radiol 2020; 30: 4356-4357.

3 Toussie D, Voutsinas N, Finkelstein M, et al. Clinical and chest radiography features determine patient outcomes in young and middle age adults with COVID-19. Radiology 2020; 297: E197-E206.

4 Richardson S, Hirsch JS, Narasimhan M, et al. Presenting characteristics, comorbidities, and outcomes among 5700 patients hospitalized with COVID-19 in the New York City Area. JAMA 2020; 323: 2052-2059.

$5 \quad$ Zhang JJY, Lee KS, Ang LW, et al. Risk factors of severe disease and efficacy of treatment in patients infected with COVID-19: a systematic review, meta-analysis and meta-regression analysis. Clin Infect Dis 2020; 71: $2199-2206$.

6 World Health Organization (WHO). WHO Coronavirus Disease (COVID-19) Dashboard https://covid19.who.int/ Date last accessed: July 31, 2020.

7 Ahmed H, Patel K, Greenwood DC, et al. Long-term clinical outcomes in survivors of severe acute respiratory syndrome and Middle East respiratory syndrome coronavirus outbreaks after hospitalisation or ICU admission: a systematic review and meta-analysis. J Rehabil Med 2020; 52: jrm00063.

8 Chiumello D, Coppola S, Froio S, et al. What's next after ARDS: long-term outcomes. Respir Care 2016; 61: 689-699.

9 Raghu G, Wilson KC. COVID-19 interstitial pneumonia: monitoring the clinical course in survivors. Lancet Respir Med 2020; 8: 839-842.

10 Huang Y, Tan C, Wu J, et al. Impact of coronavirus disease 2019 on pulmonary function in early convalescence phase. Respir Res 2020; 21: 163.

11 Williams N. The MRC breathlessness scale. Occup Med 2017; 67: 496-497.

12 Fletcher CM, Elmes PC, Fairbairn AS, et al. The significance of respiratory symptoms and the diagnosis of chronic bronchitis in a working population. Br Med J 1959; 2: 257-266.

13 Herdman M, Gudex C, Lloyd A, et al. Development and preliminary testing of the new five-level version of EQ-5D (EQ-5D-5L). Qual Life Res 2011; 20: 1727-1736.

14 van Hout B, Janssen MF, Feng YS, et al. Interim scoring for the EQ-5D-5L: mapping the EQ-5D-5L to EQ-5D-3L value sets. Value Health 2012; 15: 708-715.

15 Graham BL, Brusasco V, Burgos F, et al. 2017 ERS/ATS standards for single-breath carbon monoxide uptake in the lung. Eur Respir J 2017; 49: 1600016.

16 Graham BL, Steenbruggen I, Miller MR, et al. Standardization of spirometry 2019 update. An official American Thoracic Society and European Respiratory Society technical statement. Am J Respir Crit Care Med 2019; 200: e70-e88.

17 Quanjer PH, Stanojevic S, Cole TJ, et al. Multi-ethnic reference values for spirometry for the 3-95-yr age range: the global lung function 2012 equations. Eur Respir J 2012; 40: 1324-1343.

18 Stanojevic S, Graham BL, Cooper BG, et al. Official ERS technical standards: Global Lung Function Initiative reference values for the carbon monoxide transfer factor for Caucasians. Eur Respir J 2017; 50: 1700010.

19 Holland AE, Spruit MA, Troosters T, et al. An official European Respiratory Society/American Thoracic Society technical standard: field walking tests in chronic respiratory disease. Eur Respir J 2014; 44: 1428-1446.

20 Hansell DM, Bankier AA, MacMahon H, et al. Fleischner Society: glossary of terms for thoracic imaging. Radiology 2008; 246: 697-722.

21 Søyseth V, Aaløkken TM, Mynarek G, et al. Diagnosis of biopsy verified usual interstitial pneumonia by computed tomography. Respir Med 2015; 109: 897-903.

22 Marvisi M, Ferrozzi F, Balzarini L, et al. First report on clinical and radiological features of COVID-19 pneumonitis in a Caucasian population: factors predicting fibrotic evolution. Int J Infect Dis 2020; 99: 485-488.

23 Zhao YM, Shang YM, Song WB, et al. Follow-up study of the pulmonary function and related physiological characteristics of COVID-19 survivors three months after recovery. EClinicalMedicine 2020; 25: 100463.

24 George PM, Wells AU, Jenkins RG. Pulmonary fibrosis and COVID-19: the potential role for antifibrotic therapy. Lancet Respir Med 2020; 8: 807-815.

25 Mittl RL Jr, Schwab RJ, Duchin JS, et al. Radiographic resolution of community-acquired pneumonia. Am J Respir Crit Care Med 1994; 149: 630-635. 